\title{
Clinical characteristics and treatment propen- sity in elderly patients aged over 80 years with colorectal cancer
}

Yun Hwa Jung ${ }^{1,2}$, Jae Young Kim³ ${ }^{3}$ Yu Na Jang 3 , Sang Hoon Yoo 3 , Gyo Hui Kim³, Kang Min Lee In Kyu Lee ${ }^{4}, \mathrm{Su} \mathrm{Mi}$ Chung ${ }^{5}$, and In Sook $\mathrm{Woo}^{1}$

\begin{abstract}
${ }^{1}$ Division of Medical Oncology, Department of Internal Medicine, College of Medicine, Yeouido St. Mary's Hospital, The Catholic University of Korea, Seoul; ${ }^{2}$ Division of Hematology-Oncology, Department of Internal Medicine, Daejeon Sun Hospital, Daejeon; Departments of ${ }^{3}$ Internal Medicine, ${ }^{4}$ Surgery, and ${ }^{5}$ Radiation Oncology, College of Medicine, The Catholic University of Korea, Seoul, Korea
\end{abstract}

Received: June 22, 2016

Revised : February 12, 2017

Accepted: April 2, 2017

\section{Correspondence to}

In Sook Woo, M.D.

Division of Medical Oncol-

ogy, Department of Internal

Medicine, College of Medicine,

Yeouido St. Mary's Hospital, The

Catholic University of Korea, 10

63-ro, Yeongdeungpo-gu, Seoul

07345, Korea

Tel: $+82-2-3779-1574$

Fax: +82-2-780-3132

E-mail:insookwoo@catholic.ac.kr
Background/Aims: Elderly patients ( $\geq 80$ years) with colorectal cancer (CRC) tend to avoid active treatment at the time of diagnosis despite of recent advances in treatment. The aim of this study was to determine treatment propensity of elderly patients aged $\geq 80$ years with CRC in clinical practice and the impact of anticancer treatment on overall survival (OS).

Methods: Medical charts of 152 elderly patients (aged $\geq 80$ years) diagnosed with CRC between 1998 and 2012 were retrospectively reviewed. Patients' clinical characteristics, treatment modalities received, and clinical outcome were analyzed.

Results: Their median age was 82 years (range, 80 to 98 ). Of 152 patients, 148 were assessable for the extent of the disease. Eighty-two of 98 patients with localized disease and 28 of 50 patients with metastatic disease had received surgery or chemotherapy or both. Surgery was performed in 79 of 98 patients with localized disease and 15 of 50 patients with metastatic disease. Chemotherapy was administered in only 24 of 50 patients with metastatic disease. Patients who received anticancer treatment according to disease extent showed significantly longer OS compared to untreated patients (localized disease, 76.2 months vs. 15.4 months, $p$ $=0.000$; metastatic disease, 9.9 months vs. 2.6 months, $p=0.001$ ). Along with anticancer treatment, favorable performance status (PS) was associated with longer OS in multivariate analysis of clinical outcome.

Conclusions: Elderly patients aged $\geq 80$ years with CRC tended to receive less treatment for metastatic disease. Nevertheless, anticancer treatment in patients with favorable PS was effective in prolonging OS regardless of disease extent.

Keywords: Colorectal neoplasms; Drug therapy; Colorectal surgery; Aged

\section{INTRODUCTION}

Colorectal cancer (CRC) is one of the most commonly diagnosed cancers worldwide. Besides, the incidence of CRC tends to increase along with age. The median age at diagnosis is 70 years in developed countries. Consequently, the proportion of elderly patients with CRC is considerable and rising steadily. According to epidemiologic data from the United States, among newly diagnosed CRC patients in 2014, the proportion of patients aged $\geq 80$ years was estimated to be $19 \%$ in males and $29 \%$ in females [1]. Despite a steady increase in the number of very elderly patients aged $\geq 80$ years with CRC, prognosis of this age group is still very poor without im- 
provement, especially in patients with metastatic CRC [2]. To solve this problem, many prior studies have tried to find optimal treatment strategy in elderly CRC patients and invent practical screening tool such as comprehensive geriatric assessment to discriminate medically-fit elderly patients for anticancer treatment. In particular, International Society of Geriatric Oncology has proposed separate treatment guideline for treating elderly patients with CRC [3]. However, most previous studies that guide our current treatment of elderly patients with CRC did not include many patients over 80 years of age. In addition, there is discrepancy between suggested treatment guideline and 'real' clinical practice. In this respect, investigating how this age group of patients is treated in 'real' clinical practice and what is the problem in treating them is of great clinical significance. Based on a few studies that investigated treatment status of elderly patients with CRC, there was a distinct difference in treatment propensity between patients under 80 years and over 80 years old. One particularly striking difference that should be addressed was the under-treatment propensity in elderly patients aged $\geq 80$ years $[4,5]$. Usually, as patients get older (especially those aged $\geq 8$ o years), anticancer treatment tends to be waived for the following reasons: (1) it is thought that anticancer treatment might worsen quality of life (QOL) of patients because of complications and treatment related toxicities; (2) survival benefits of anticancer treatment in elderly patients are uncertain because of the relatively shorter life expectancy associated with old age itself; (3) comorbid illness of elderly patients may lead vulnerable patients into worse general health status following active anticancer therapy; and (4) lack of economic rights and caring in the family compared to younger patients could be an obstacle to active anticancer treatment. However, some studies have shown that proper anticancer treatment for selected patients could achieve survival benefits even for very elderly patients aged $\geq 8$ o years old [6]. Therefore, more clinical studies specifically aiming at this age group are required. Therefore, the objective of this study was to retrospectively review medical records of very elderly patients aged $\geq 8$ o years old with CRC to determine treatment propensity and clinical outcomes.

\section{METHODS}

\section{Study design and statistical analysis}

Clinical records of 152 consecutive patients (aged $\geq 80$ years) who were diagnosed with CRC between 1998 and 2012 were retrospective reviewed. Clinical characteristics and treatment modalities of all 152 patients along with clinical outcomes were analyzed. Specifically, the following patient characteristics were analyzed: age, sex, tumor location, stage at presentation defined by American Joint Committee on Cancer (AJCC) system, number of comorbid illnesses, Eastern Cooperative Oncology Group (ECOG) performance status (PS), serum carcinoembryonic antigen (CEA) level, and body mass index (BMI) on diagnosis.

For treatment-related factors, initial treatment modalities (especially focused on surgery and chemotherapy) received by each patient and their purpose were analyzed. In case of chemotherapy, purpose (adjuvant vs. palliative), chemotherapy intensity (monotherapy vs. combination), and drug administrated were determined.

Because principal treatment strategy (surgery vs. chemotherapy) depends on disease extent, patients were divided into two groups by disease extent (localized disease [stage I-III] vs. metastatic disease [stage IV]) for further analysis. Availability of each treatment modality (especially surgery and chemotherapy) and clinical factors potentially affecting treatment availability in different groups were investigated. In particular, surgical treatment in localized disease and chemotherapy in metastatic disease were analyzed. In case of patients who did not receive any anti-cancer treatment except for palliative chemotherapy, the reason of non-treatment was investigated by chart review.

In addition, safety profile of each anticancer treatment modality was investigated. In case of surgical treatment, 6o-day postoperative mortality rate, cause of death, and postoperative complication rate were analyzed. In case of chemotherapy, duration of treatment and cause of interruption were analyzed. Lastly, the impact of anticancer treatment on overall survival (OS) and clinical characteristics related to prognosis were investigated according to the extent of disease (localized vs. metastatic).

Statistical analysis was performed with SPSS version 18.0 (SPSS Inc., Chicago, IL, USA). p values of < 0.05 were 
considered statistically significant. OS was defined from the date of initial diagnosis to the date of death. The Kaplan-Meier method was used to analyze OS. Log rank test was used to compare differences in survival between groups. Independent-sample $t$ test and chi-square test were used to compare treatment pattern according to clinical characteristics and extent of disease (localized vs. metastatic). Univariate analysis was performed to assess which factors impacted OS. Variables that were found to be significant $(p<0.05)$ in univariate analysis were entered into multivariate logistic regression analysis. The final model was chosen with significant $(p<$ 0.05) variables.

\section{Ethical approval}

This study was conducted with the permission of the Institutional Review Board of Yeouido St. Mary's Hospital (SC16RISIoo48). Because this study was a retrospective medical record-based study, the IRB waved the need for written consent from the patients.

\section{RESULTS}

\section{Patient characteristics}

A total of 152 elderly patients (aged $\geq 80$ years) were investigated. There were 75 males (49.3\%) and 77 females (50.7\%). Their median age was 82 years (range, 80 to 98 ). One hundred six and 46 patients were diagnosed with colon cancer and rectal cancer, respectively. Ninetyeight patients $(64.5 \%)$ were at localized stage (I-III) and 50 patients $(32.9 \%)$ were in stage IV. The stages of four patients $(2.6 \%)$ could not be assessed because of incomplete evaluation. A total of 128 patients $(84.2 \%)$ had ECOG performance status (PS) of 2 or less. A total of 104 patients $(68.4 \%)$ presented with at least one comorbidity, including hypertension (40.1\%) or diabetes (19.7\%), while 49 patients $(32.2 \%)$ had more than two comorbid illnesses (Table 1).

\section{Treatment}

Physician considered carefully the will of patients and their family before the decision of treatment (Fig. 1). At the time of initial diagnosis, 111 patients (73\%) received anticancer treatment with surgery, chemotherapy, or multimodalities. On the other hand, 41 patients (27\%)
Table 1. Clinical characteristics of all patients $(n=152)$

\begin{tabular}{|c|c|}
\hline Clinical characteristic & No. (\%) \\
\hline Age, yr, median (range) & $82(80-98)$ \\
\hline $80-84$ & $109(71.7)$ \\
\hline $85-89$ & $30(19.7)$ \\
\hline$\geq 90$ & $13(8.6)$ \\
\hline \multicolumn{2}{|l|}{ Sex } \\
\hline Male & $75(49 \cdot 3)$ \\
\hline Female & $77(50.7)$ \\
\hline \multicolumn{2}{|l|}{ Tumor location } \\
\hline Colon & $106(69.7)$ \\
\hline Ascending colon & $41(27.0)$ \\
\hline Transverse colon & $7(4.6)$ \\
\hline Descending/sigmoid colon & $58(38.1)$ \\
\hline Rectum & $46(30.3)$ \\
\hline \multicolumn{2}{|l|}{ Clinical stage } \\
\hline I & $31(20.4)$ \\
\hline II & $32(21.1)$ \\
\hline III & $35(23.0)$ \\
\hline IV & $50(32.9)$ \\
\hline Missing & $4(2.6)$ \\
\hline \multicolumn{2}{|l|}{ Performance status } \\
\hline 1 & $98(64.5)$ \\
\hline 2 & $30(19.7)$ \\
\hline 3 & $22(14 \cdot 5)$ \\
\hline 4 & $2(1.3)$ \\
\hline \multicolumn{2}{|l|}{ Initial CEA } \\
\hline Normal & $67(44.1)$ \\
\hline Elevated & $58(38.1)$ \\
\hline Unavailable & $27(17.8)$ \\
\hline \multicolumn{2}{|l|}{$\mathrm{BMI}, \mathrm{kg} / \mathrm{m}^{2}$} \\
\hline$<20$ & $24(15.8)$ \\
\hline$\geq 20$ and $<25$ & $61(40.1)$ \\
\hline$\geq 25$ & $25(16.5)$ \\
\hline Missing & $42(27.6)$ \\
\hline \multicolumn{2}{|l|}{ Comorbidities } \\
\hline Hypertension & $61(40.1)$ \\
\hline Diabetes & $30(19.7)$ \\
\hline Cerebrovascular disease & $10(6.6)$ \\
\hline Other malignancies & $11(7.2)$ \\
\hline \multicolumn{2}{|l|}{ No. of comorbidities } \\
\hline No disease & $48(31.6)$ \\
\hline 1 Disease & $55(36.2)$ \\
\hline$\geq 2$ Diseases & $49(32.2)$ \\
\hline
\end{tabular}

CEA, carcinoembryonic antigen; BMI, body mass index. 
152 Total elderly (aged $\geq 80$ ) colorectal cancer patients

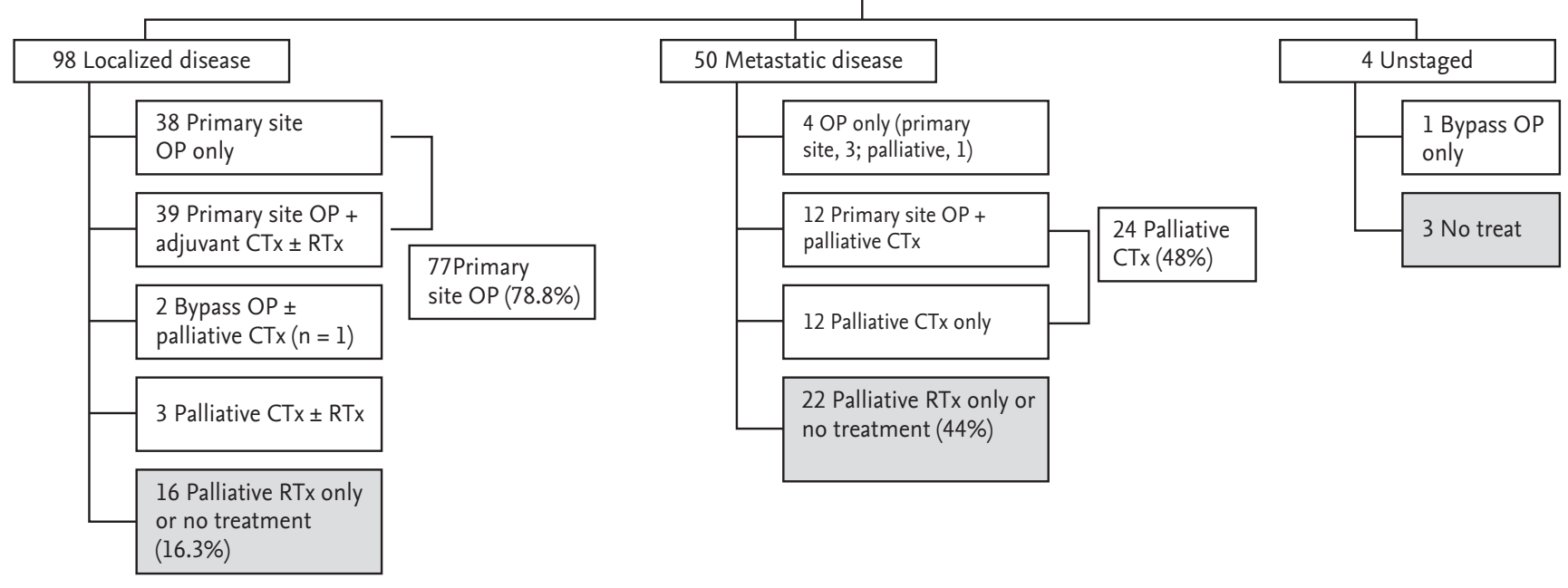

92 Primary site OP

4 Bypass surgery

39 Adjuvant CTx

28 Palliative $C T x$

Figure 1. Summary of received initial treatment modalities in very elderly colorectal cancer patients. CTx, chemotherapy; OP, operation; RTx, radiation therapy.

received only the best supportive care or palliative radiation therapy for symptomatic relief. Classified by disease extent, 16 of 98 patients $(16.3 \%)$ with localized disease and 22 of 50 patients (44\%) with metastatic disease did not receive surgery or chemotherapy. Although not all causes of treatment refusal could be assessed due to missing data, the most common cause of no-treatment was due to refusal by patients or their families (21/41), followed by poor PS caused by comorbidities (5/41), death by cancer related complications (4/41), and economic difficulty $(1 / 41)$. Notably, the proportion of patients who refused anticancer treatment was higher in patients with metastatic disease (12/22) than that in patients with localized disease $(6 / 16)$.

\section{Surgery}

A total of 96 surgeries were performed, including 92 primary site resections and four bypass surgeries only. Primary site resection was performed in 77 of 98 patients (78.6\%) with localized disease (stage I, 25/31; stage II, 28/32; stage III, 24/35). Among the remaining 21 patients, two patients received only bypass surgery without primary site resection for palliative purposes. Primary site resection was performed in 15 of 50 patients (30\%) with metastatic disease. Among them, two patients received metastatectomy for liver metastasis. Of four unstaged patients, one patient received palliative bypass surgery without primary site resection.

\section{Chemotherapy}

Chemotherapy availability was investigated according to treatment purpose. Adjuvant chemotherapy was performed in 39 of 77 patients who received curative intent surgery for localized disease. Among patients at stage III, 75\% (18/24) received adjuvant chemotherapy after curative surgery. After analyzing prescribed treatment regimen, most patients $(33 / 39,84.6 \%)$ received oral agents such as 5'-deoxy-5-fluorouridine (doxifluridine, 600 to $1,200 \mathrm{mg} /$ day, $\mathrm{n}=26$ ), tegafur-uracil (UFT, $300 \mathrm{mg} / \mathrm{m}^{2} /$ day for 28 days every 5 weeks, $\mathrm{n}=1$ ), Polysaccharide kureha (PSK; $3 \mathrm{~g} /$ day, $\mathrm{n}=10$ ), or capecitabine $\left(2,000 \mathrm{mg} / \mathrm{m}^{2} /\right.$ day for 14 days every 3 weeks, $n=5$ ) in adjuvant setting. PSK was prescribed in monotherapy $(n=1)$ or combination ( $\mathrm{n}=9$ ) with other oral chemotherapeutic agents. Six patients (15.4\%) received intravenous (IV) drugs for adjuvant chemotherapy. All these patients received a fluorouracil (5-FU)/leucovorin (LV) regimen.

As for palliative setting, 24 of 50 patients (48\%) who 
had metastatic disease received first-line chemotherapy. Prescribed chemotherapeutic agents were doxifluridine, capecitabine, 5-FU/LV, oxaliplatin, and irinotecan. Of the 24 patients, $16(66.6 \%)$ received monotherapy while 8 (33.3\%) received combination chemotherapy. With regard to administration route, 14 patients received oral agents while 10 patients received IV drugs. Of the 10 patients who received IV drugs, eight received combination chemotherapy (oxaliplatin-containing regimen in seven patients and irinotecan-containing regimen in one patient). Of 24 patients who received first-line palliative chemotherapy, only six $(25 \%)$ received second-line treatment after progression. Among them, two patients eventually received fluorouracil, oxaliplatin, and irinotecan throughout the treatment course. A targeted agent (cetuximab plus irinotecan) was used in one patient as a second-line treatment.

\section{Treatment propensity analysis}

When treatment availability was compared according to disease extent, the first noticeable finding was a disparity of treatment availability between localized disease and metastatic disease (Table 2). Surgical treatment availability (80.6\%) for patients with localized disease was higher than chemotherapy availability (48\%) for patients with metastatic disease.

Next, various clinical factors expected to influence surgical treatment availability in localized disease and chemotherapy availability in metastatic disease were analyzed. The proportion of patients who received surgery or chemotherapy in the respective cohort (limited disease vs. metastatic disease) was not significantly affected by age subcategory ( 80 to 84,85 to 89 , and $\geq 90$ ), sex, serum CEA level, or number of comorbidities. However, patients with better PS more frequently received surgery $(p=0.001)$ or chemotherapy $(p=0.012)$ with statistical significance. In addition, patients with higher BMI $(\geq 20)$ were more likely to receive surgery, whereas patients with rectal cancer were less likely to received surgery (Table 2).

\section{Safety profile}

In 96 patients who underwent surgery, postoperative complications occurred in 27 patients $(28.1 \%)$, including ileus ( $(n=6)$, delirium $(n=4)$, pneumonia $(n=4)$, wound infection $(n=4)$, cardiac complications $(n=3$, includ- ing two arrhythmia and one heart failure), bleeding ( $\mathrm{n}$ $=2)$, ulcer $(n=1)$, sepsis $(n=1)$, cerebral infarct $(n=1)$, and urologic complication $(\mathrm{n}=1)$. Post-operation 6oday mortality was $6.25 \%$ (6/96) for the total number of patients who received surgery. All six patients who deceased within 60 days after surgery had received emergency operations because of obstruction (two patients) or perforation (four patients). Causes of post-operative death were pneumonia $(\mathrm{n}=3)$, cardiac complication $(\mathrm{n}=$ 2), and $\operatorname{sepsis}(\mathrm{n}=1)$.

Regarding chemotherapy, among 33 patients who received oral agents as adjuvant chemotherapy, 28 patients $(84.8 \%)$ continued treatment over 6 months or six cycles. One patient who received adjuvant capecitabine monotherapy interrupted the treatment after the 3 rd cycle because of renal impairment and deterioration of PS. Three patients who received doxifluridine refused to continue adjuvant chemotherapy before 6 months due to gastrointestinal side effect. One patient who received PSK stopped treatment after 3 months due to shortage of necessity. All six patients who received IV 5-FU/LV regimen as adjuvant chemotherapy completed the initially intended six cycles of chemotherapy.

Among 24 patients who received palliative chemotherapy, eight (monotherapy, $\mathrm{n}=4$; combination therapy, $\mathrm{n}=$ 4) stopped the treatment without progression due to side effect or deterioration of general condition. Most common cause of treatment interruption was general weakness rather than specific treatment related complications such as hematologic toxicity or mucositis. Especially, two patients (one on doxifluridine and one on 5 -FU/LV) expired during treatment due to pneumonia and disseminated intravascular coagulation, respectively.

\section{Treatment outcome}

The efficacy of anticancer treatment was analyzed by survival analysis. Because of paucity of cases and poor rate of response evaluation, other outcome related factors such as recurrence rate or response rate were not analyzed. OS was analyzed according to anticancer treatment in two groups with different disease extent (localized vs. metastatic disease). The median OS was significantly different between treated patients and untreated patients in both localized and metastatic diseases (localized disease, 76.2 months vs. 15.4 months, $p=0.000$; metastatic disease, 9.9 months vs. 2.6 months, $p=0.001$ ) (Fig. 
$2 \mathrm{~A}$ and $2 \mathrm{~B}$ ). Additionally, we compared OS according to the number of chemotherapeutic agents (monotherapy vs. combination therapy). The OS of patients treated with monotherapy was not inferior to that of patients treated with combination chemotherapy (Fig. 2C). Our analysis also explored clinical factors associated with OS in each cohort (localized disease or metastatic disease).
On univariate analysis of prognostic factors in patients' group with localized disease, the median OS was significantly shorter in patients with male gender, poor PS (ECOG 3-4), no anticancer treatment (in particular, operation), and lower BMI (Table 3). However, tumor location, age, adjuvant chemotherapy, serum CEA level, or number of comorbid illnesses was not significant-

Table 2. Comparison of treatment propensity according to clinical characteristics and disease extent $(n=148)$

\begin{tabular}{|c|c|c|c|c|c|c|c|}
\hline \multirow[b]{2}{*}{ Characteristic } & \multicolumn{3}{|c|}{ Stage I-III $(n=98)$} & \multicolumn{4}{|c|}{ Stage IV $(\mathrm{n}=50)$} \\
\hline & $\begin{array}{c}\text { Surgery } \\
(\mathrm{n}=79,80.6 \%)\end{array}$ & $\begin{array}{c}\text { No surgery } \\
(\mathrm{n}=19,19.4 \%)\end{array}$ & $p$ value $^{a}$ & $\begin{array}{c}\text { No CTx } \\
(n=26,52 \%)\end{array}$ & $\begin{array}{c}\text { Mono CTx } \\
(n=16,32 \%)\end{array}$ & $\begin{array}{c}\text { Combi CTx } \\
(\mathrm{n}=8,16 \%)\end{array}$ & $p$ value ${ }^{a}$ \\
\hline Age, yr & & & 0.810 & & & & 0.126 \\
\hline $80-84$ & $60(75.9)$ & $14(73.7)$ & & $16(61.5)$ & $10(62.5)$ & $7(87.5)$ & \\
\hline $85-89$ & $14(17 \cdot 7)$ & $3(15.8)$ & & $5(19.2)$ & $6(37 \cdot 5)$ & $1(12.5)$ & \\
\hline$\geq 90$ & $5(6.3)$ & $2(10.5)$ & & $5(19.2)$ & $\mathrm{O}$ & o & \\
\hline Sex & & & 0.111 & & & & 0.132 \\
\hline Male & $38(48.1)$ & $13(68.4)$ & & $9(34.6)$ & $9(43.8)$ & $6(75.0)$ & \\
\hline Female & $41(51.9)$ & $6(31.6)$ & & $17(65 \cdot 4)$ & $7(56.3)$ & $2(25.0)$ & \\
\hline Tumor location & & & $0.002^{b}$ & & & & 0.155 \\
\hline Ascending colon & $22(27.8)$ & $1(5 \cdot 3)$ & & $7(26.9)$ & $8(50.0)$ & $3(37 \cdot 5)$ & \\
\hline Transverse colon & $4(5 \cdot 1)$ & $1(5 \cdot 3)$ & & $1(3.8)$ & 0 & 0 & \\
\hline Descending/sigmoid colon & $35(44 \cdot 3)$ & $4(21.1)$ & & $7(26.9)$ & $5(31.3)$ & $5(62.5)$ & \\
\hline Rectum & $17(21.5)$ & $13(68.4)$ & & $11(42.3)$ & $3(18.8)$ & 0 & \\
\hline No. of comorbidities & & & 0.172 & & & & 0.444 \\
\hline No disease & $23(29.1)$ & $3(15.8)$ & & $13(50.0)$ & $6(37 \cdot 5)$ & $1(12.5)$ & \\
\hline 1 Disease & $32(40.5)$ & $6(31.6)$ & & $7(26.9)$ & $6(37.5)$ & $4(50.0)$ & \\
\hline$\geq 2$ Diseases & $24(30.4)$ & $10(52.6)$ & & $6(23.1)$ & $4(25.0)$ & $3(37 \cdot 5)$ & \\
\hline Performance status & & & $0.001^{b}$ & & & & $0.012^{b}$ \\
\hline $0-1$ & $61(77.2)$ & $7(36.8)$ & & $10(38.5)$ & $12(75 \cdot 0)$ & $7(87.5)$ & \\
\hline $2-4$ & $18(22.8)$ & $12(63.2)$ & & $16(61.5)$ & $4(25 \cdot 0)$ & $1(12.5)$ & \\
\hline BMI, $\mathrm{kg} / \mathrm{m}^{2}$ & & & 0.010 & & & & 0.856 \\
\hline$<20$ & $8(10.1)$ & $5(26.3)$ & & $6(23.1)$ & $3(18.8)$ & $2(25.0)$ & \\
\hline$\geq 20$ and $<25$ & $40(50.6)$ & $4(21.1)$ & & $7(26.9)$ & $6(37 \cdot 5)$ & $4(50.0)$ & \\
\hline$\geq 25$ & $19(24.1)$ & $1(5 \cdot 3)$ & & $2(7 \cdot 7)$ & $1(6.2)$ & $2(25 \cdot 0)$ & \\
\hline Missing & $12(15 \cdot 2)$ & $9(47 \cdot 4)$ & & $11(42.3)$ & $6(37.5)$ & 0 & \\
\hline Initial CEA & & & 0.321 & & & & 0.237 \\
\hline Normal & $51(64.6)$ & $9(47 \cdot 3)$ & & $1(3.8)$ & $4(25.0)$ & $1(12.5)$ & \\
\hline Elevated & $19(24.0)$ & $6(31.6)$ & & $15(57 \cdot 7)$ & $10(62.5)$ & $7(87.5)$ & \\
\hline Missing & $9(11.4)$ & $4(21.1)$ & & $10(38.5)$ & $2(12.5)$ & 0 & \\
\hline
\end{tabular}

Values are presented as number (\%).

CTx, chemotherapy; BMI, body mass index; CEA, carcinoembryonic antigen.

${ }^{a}$ The comparison of variables between each treatment modality within each cohort (chi-square test).

${ }^{\mathrm{b}} \mathrm{p}<0.05$. 

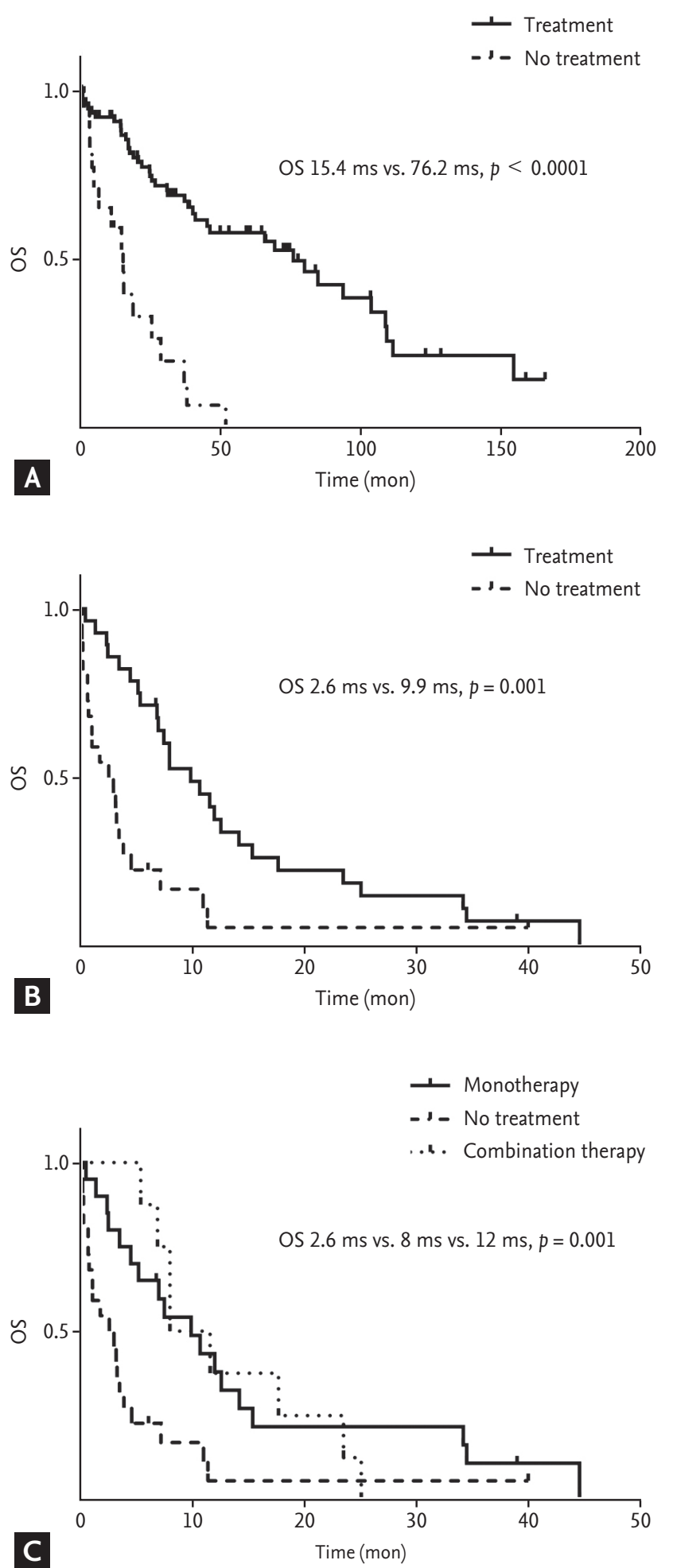

Figure 2. Kaplan-Meier plots of overall survival (OS) for elderly patients with localized colorectal cancer (CRC) (A) and metastatic CRC (mCRC) (B) according to treatment pattern. (C) Kaplan-Meier plots of OS for elderly patients with mCRC according to number of anticancer agents (monotherapy vs. combination regimen). ly related to survival duration. On univariate analysis of prognostic factors in patients' group with metastatic disease, poor PS and no anticancer treatment (both operation and chemotherapy) were significantly related to poor prognosis. Multivariate analysis was separately performed according to disease extent with significant factors identified from univariate analysis. In patients with localized disease, good PS and surgery were significantly related to longer OS. On the other hand, in patients with advanced disease, palliative chemotherapy and favorable PS resulted in longer OS with statistical significance (Table 4).

\section{DISCUSSION}

Along with increase of elderly patients with CRC, many clinical studies on the feasibility and outcomes of anticancer treatment in elderly patients with CRC have been reported. However, most previous studies defined the cut-off age of 'elderly' patients as $\geq 65$ or 70 years old. There have not been many studies focusing on patients aged more than 80 years old. Therefore, clinicians had to depend on clinical studies of elderly patients primarily in their sixties or seventies to decide treatment strategy for octogenarians or older patients with CRC. Consequently, there had been disparity between clinical guideline and 'real' practice in the treatment of elderly CRC patients aged over 80 years old.

With regard to surgical treatment, many recent studies have demonstrated that patients aged over 80 years old and even nonagenarians could achieve favorable outcomes with operation except for emergent cases [79]. Based on these results, surgical treatment has been actively encouraged for elderly CRC patients aged $\geq 80$ years. Consequently, the gap between recommended treatment guideline and 'real' practice has been narrowed in surgical management of very elderly CRC patients. Indeed, according to a few recent population-based studies showing current treatment status of elderly patients with CRC, the proportion of very elderly patients aged over 80 years old receiving surgery is increased at a level similar to that in young patients $[10,11]$. Our institution also actively performed surgery for the majority (79/98, 80.6\%) of very elderly CRC patients aged $\geq 80$ years with localized disease (stage I-III) during the 
Jung YH, et al. Treatment of very elderly CRC patients

Table 3. Prognostic factor for overall survival according to disease extent in univariate analysis (log rank test)

\begin{tabular}{|c|c|c|c|c|c|c|}
\hline \multirow[b]{2}{*}{ Characteristic } & \multicolumn{3}{|c|}{ Localized disease } & \multicolumn{3}{|c|}{ Systemic disease } \\
\hline & $\begin{array}{l}\text { Number } \\
(\mathrm{n}=98)\end{array}$ & $\begin{array}{l}\text { Median OS, } \\
\text { ms }\end{array}$ & $\begin{array}{c}\text { Univariate } \\
p \text { value }\end{array}$ & $\begin{array}{l}\text { Number } \\
(\mathrm{n}=50)\end{array}$ & $\begin{array}{l}\text { Median OS, } \\
\text { ms }\end{array}$ & $\begin{array}{l}\text { Univariate } \\
p \text { value }\end{array}$ \\
\hline Age, yr & & & 0.230 & & & 0.517 \\
\hline $80-84$ & 74 & 52.0 & & 33 & $5 \cdot 4$ & \\
\hline $85-89$ & 17 & 26.8 & & 12 & 7.2 & \\
\hline$\geq 90$ & 7 & 93.9 & & 5 & 3.9 & \\
\hline Sex & & & $0.018^{\mathrm{a}}$ & & & 0.617 \\
\hline Female & 47 & 103.9 & & 28 & $4 \cdot 5$ & \\
\hline Male & 51 & 37.2 & & 22 & 7.0 & \\
\hline Primary tumor site & & & 0.226 & & & 0.144 \\
\hline Ascending colon & 23 & $45 \cdot 4$ & & 18 & $7 \cdot 5$ & \\
\hline Transverse colon & 5 & 24.8 & & 1 & 1.1 & \\
\hline Descending colon & 39 & $65 \cdot 9$ & & 17 & 7.2 & \\
\hline Rectum & 31 & 25.6 & & 14 & $3 \cdot 5$ & \\
\hline Performance status (ECOG) & & & $0.000^{\mathrm{a}}$ & & & $0.000^{\mathrm{a}}$ \\
\hline $0-1$ & 68 & 84.9 & & 29 & 11.0 & \\
\hline $2-4$ & 30 & 14.8 & & 21 & 2.6 & \\
\hline \multicolumn{7}{|l|}{ Treatment } \\
\hline Cancer treatment & & & $0.000^{\mathrm{a}}$ & & & $0.001^{\mathrm{a}}$ \\
\hline Yes & 81 & 76.2 & & 28 & 9.9 & \\
\hline No & 17 & $15 \cdot 4$ & & 22 & 2.6 & \\
\hline Operation & & & $0.000^{\mathrm{a}}$ & & & $0.017^{\mathrm{a}}$ \\
\hline Yes & 79 & 80.2 & & 16 & 10.7 & \\
\hline No & 19 & $15 \cdot 4$ & & 34 & $3 \cdot 5$ & \\
\hline Chemotherapy & \multicolumn{2}{|c|}{ Adjuvant } & 0.109 & \multicolumn{2}{|c|}{ Palliative } & $0.000^{\mathrm{a}}$ \\
\hline Yes & 42 & 65.9 & & 24 & 11.6 & \\
\hline No & 56 & 38.2 & & 26 & 2.6 & \\
\hline Initial CEA & & & 0.119 & & & 0.736 \\
\hline Normal & 60 & 69.5 & & 6 & 6.9 & \\
\hline Elevated & 25 & 31.3 & & 32 & 7.2 & \\
\hline Unavailable & 13 & & & 12 & & \\
\hline $\mathrm{BMI}, \mathrm{kg} / \mathrm{m}^{2}$ & & & $0.000^{a}$ & & & 0.402 \\
\hline$<20$ & 13 & 24.8 & & 11 & 4.5 & \\
\hline$\geq 20$ and $<25$ & 44 & 69.5 & & 17 & 11.0 & \\
\hline$\geq 25$ & 20 & 109.1 & & 5 & 8.0 & \\
\hline Unavailable & 21 & & & 17 & & \\
\hline Comorbidities & & & 0.620 & & & 0.831 \\
\hline No disease & 26 & $65 \cdot 9$ & & 20 & $5 \cdot 4$ & \\
\hline 1 Comorbid & 38 & 31.0 & & 17 & $5 \cdot 2$ & \\
\hline$\geq 2$ Comorbid & 34 & 38.2 & & 13 & 6.9 & \\
\hline Unavailable & & & & & & \\
\hline
\end{tabular}

OS, overall survival; ECOG, Eastern Cooperative Oncology Group; CEA, carcinoembryonic antigen; BMI, body mass index. $\mathrm{a} p<0.05$. 
Table 4. Prognostic factor for overall survival in multivariate analysis

\begin{tabular}{|c|c|c|c|}
\hline \multirow{2}{*}{ Variable } & \multicolumn{3}{|c|}{ Multivariate } \\
\hline & HR & $95 \% \mathrm{CI}$ & $p$ value \\
\hline \multicolumn{4}{|l|}{ Localized disease $(n=98)$} \\
\hline ECOG PS $(2-4)$ & 5.697 & $2.615-12.415$ & $0.000^{a}$ \\
\hline Male patients & 2.055 & $0.869-4.860$ & 0.101 \\
\hline Operation & 0.270 & $0.111-0.658$ & $0.004^{\mathrm{a}}$ \\
\hline Increased BMI $\left(\geq 20 \mathrm{~kg} / \mathrm{m}^{2}\right)$ & 0.509 & $0.233-1.110$ & 0.090 \\
\hline \multicolumn{4}{|l|}{ Metastatic disease $(n=50)$} \\
\hline ECOG PS (2-4) & 3.356 & $1.677-6.716$ & $0.001^{\mathrm{a}}$ \\
\hline Chemotherapy & 0.479 & $0.232-0.988$ & $0.046^{a}$ \\
\hline Operation & 0.509 & $0.237-1.092$ & 0.083 \\
\hline
\end{tabular}

HR, hazard ratio; CI, confidence interval; ECOG, Eastern Cooperative Oncology Group; PS, performance status; BMI, body mass index.

${ }^{\mathrm{a}} \mathrm{p}<0.05$.

last decade. Even in patients with metastatic disease, primary site resection was performed in a significant proportion (15/50, 30\%). Outcomes of surgery were also good in despite of concerns about treatment-related mortality (TRM) in frail elderly patients. In our data, 60day postoperative mortality was $6.25 \%$ (6/96) and all 6 patients were cases of emergency surgery.

Many elderly CRC patients in their eighties or older age are less likely to receive chemotherapy. According to a few large population based studies analyzing treatment patterns of CRC patients in a national cohort, among patients with metastatic CRC, palliative chemotherapy is only given to a minor portion (28\% to $48 \%$ ) of old age group (aged $\geq 75$ years) while most ( $68 \%$ to $85 \%$ ) patients in the younger age group (aged $<75$ years) have received palliative chemotherapy $[12,13]$. This was in contrast to the finding of this study showing that surgery was performed almost equally in both young and old age group of patients with localized disease at the rate of more than $80 \%$. Our study also showed a pattern of inferior chemotherapy availability. However, adjuvant chemotherapy availability (75\%) of patients with stage III CRC was higher than palliative chemotherapy availability (48\%). More prominent under-treatment tendency in metastatic setting has also been observed in other previous studies [12-14]. This finding indicates that the cause of low availability of systemic chemotherapy in very elderly patients with metastatic disease is not just poor tolerability or discomfort to chemotherapy. Findings of this study will help explain the cause of under-treatment for elderly patients with metastatic disease and provide some tips to overcome such problem.

When characteristics according to treatment availability on surgery and chemotherapy were compared, PS was significantly better in patients who received treatment than that in patients who had no-treatment regarding both treatment modalities. Considering that disease extent can affect PS, under-treatment of patients with metastatic disease compared to patients with localized disease can be taken for granted. However, based on our result, 18 of 30 patients with unfavorable PS received surgical treatment in the localized disease group. On the other hands, only five of 21 patients with unfavorable PS received palliative chemotherapy in the metastatic disease group. Surprisingly, among 29 patients with favorable PS (o-1), 10 patients did not receive systemic chemotherapy in the metastatic disease group. The most common cause of non-treatment was refusal by patients themselves or their families rather than physicians. Refusal of treatment by patients or patients' families was more common in patients with metastatic disease than that in patients with localized disease. Therefore, patients' and caregivers' attitudes have significant impact on under-treatment propensity of metastatic CRC patients.

It has been reported that the fatalism of patients or their families may play a role in the decision-making process in elderly patients [6]. Consequently, assessing 
patients' emotional status and evidence-based recommendations about potential survival gains from anticancer treatment and reassurance of low treatment-related toxicity will be helpful for overcoming under-treatment tendency in elderly patients with CRC.

In terms of treatment related factors, our results showed that most patients received monotherapy regimen as systemic treatment, especially 5 -FU both in adjuvant setting (100\%) and palliative setting (66.6\%) in very elderly CRC patients aged over 80 years old. Until recently, 5-FU monotherapy has played a central role in treating elderly patients with metastatic CRC. The number of patients receiving single-agent 5-FU or capecitabine as first-line chemotherapy still remains high in 'real' clinical practice [15]. Another feature of systemic chemotherapy for very elderly patients in our results was that oral agents were frequently prescribed in both adjuvant setting (84.6\%) and palliative setting (66.6\%). In the early 2000 , equivalent efficacy of oral fluoropyrimidine compared to IV 5 -FU was reported in a few pivotal clinical studies $[16,17]$. In terms of toxicity, oral agents were more favorable. For that reason, oral agents including capecitabine and UFT have been used by elderly patients. The usage of oral agent has been estimated to increase in referred patients receiving palliative chemotherapy in Western countries $[18,19]$. In the same context, the higher proportion of patients treated with 5 -FU monotherapy as oral agent in adjuvant setting might explain the higher chemotherapy availability of adjuvant setting than palliative treatment. Although a few wellknown facilities have recently proposed reduced-dose FOLFOX or FOLFIRI combination chemotherapy as an initial treatment for robust elderly patients for young patients in palliative setting [20], administering intensive combination chemotherapy to very elderly patients (aged over 80 years old) with relatively limited life expectancies remains controversial. Whether better response rates with combination chemotherapy are essentially connected to longer OS is uncertain and deterioration of QOL caused by treatment-related toxicity cannot be ignored [21]. In our results, half of patients (4/8) who received combination regimen as systemic chemotherapy stopped treatment because of general weakness or deterioration of QOL even though only selected patients received chemotherapy and almost all patients received reduced dose of chemotherapy upfront. Moreover, OS of patients treated with combination chemotherapy was not superior to that of patients treated with monotherapy in the metastatic disease group. Recently, a few studies have demonstrated that doublet front-line chemotherapy provides no survival benefit in elderly CRC patients [22], supporting our results. In contrast, most patients who received monotherapy as adjuvant chemotherapy or palliative chemotherapy barely experienced significant toxicity or treatment related death. In this respect, fluoropyrimidine monotherapy (particularly oral agents such as DFUR or capecitabine) can be considered as reasonable choice of systemic chemotherapy for very elderly patients aged $\geq 8$ o years with CRC.

Another important feature of very elderly patients on chemotherapy was identified while reviewing our study results. That is, they showed poor medication adherence. This was a main drawback of oral chemotherapeutic agents. Adherence is crucial for the success of oral anticancer agent therapy, especially in ambulatory practice. A few clinical studies have investigated adherence rates for oral and IV anticancer agents in patients with CRC. It has been demonstrated that adherence with IV chemotherapy is higher compared to oral agents. In addition, adherence rate of oral agents is significantly lower in patients aged $\geq 80$ years compared to that in younger patients $[23,24]$. Treatment persistence was also found to be low in elderly patients of this study. Identification of potential predictors of poor adherence would facilitate the utilization of oral anticancer agents and improve clinical outcomes of elderly CRC patients. Moreover, meticulous supervision with proper education and precise monitoring of responses and adherence are needed to estimate the genuine efficacy of oral chemotherapeutic agents in very elderly patients with CRC.

Lastly, more randomized prospective studies are warranted to elucidate optimal single oral agent, including new drugs such as TS-1 or TAS-102 [25]. At the same time, more clinical studies on efficacy and tolerability of altered administration schedule of oral chemotherapeutic agents such as metronomic chemotherapy are needed to determine treatment option for very elderly CRC patients $[26,27]$.

Unfortunately, our study did not include many patients who received biologic agents. However, many clinical trials using combination regimens including biologic agents such as cetuximab and bevacizumab have 
been tried in elderly patients with CRC $[28,29]$. Consistent attention to clinical outcomes and safety profiles of these biologic agents in elderly patients is required.

In conclusion, our results showed that very elderly patients aged $\geq 80$ years with CRC tended to be deprived of palliative chemotherapy while a relatively significant portion of elderly patients with localized CRC received surgery and adjuvant chemotherapy. However, palliative chemotherapy in selected patients with good PS was obviously associated with improved OS. Considering beneficial effects of chemotherapy, more active treatment approaches with low toxicity profiles need to be considered for medically fit elderly patients aged over 80 years old. Prospective randomized clinical trial is required to determine the optimal tailored treatment for elderly patients aged $\geq 80$ years with CRC in the aging society and an era of precision medicine.

\section{KEY MESSAGE}

1. Very elderly patients aged $\geq 80$ years with colorectal cancer (CRC) tended to be deprived of palliative chemotherapy while a relatively significant portion of elderly patients with localized CRC received surgery.

2. Palliative chemotherapy in selected elderly patients with good performance status was obviously associated with improved overall survival.

3. Oral fluoropyrimidine monotherapy in palliative setting could be a reasonable option for very elderly CRC patients.

\section{Conflict of interest}

No potential conflict of interest relevant to this article was reported.

\section{REFERENCES}

1. Siegel R, Desantis C, Jemal A. Colorectal cancer statistics, 2014. CA Cancer J Clin 2014;64:104-117.

2. Sorbye H, Cvancarova M, Qvortrup C, Pfeiffer P, Glimelius B. Age-dependent improvement in median and longterm survival in unselected population-based Nordic registries of patients with synchronous metastatic colorectal cancer. Ann Oncol 2013;24:2354-2360.

3. Papamichael D, Audisio RA, Glimelius B, et al. Treatment of colorectal cancer in older patients: International Society of Geriatric Oncology (SIOG) consensus recommendations 2013. Ann Oncol 2015;26:463-476.

4. Bojer AS, Roikjaer O. Elderly patients with colorectal cancer are oncologically undertreated. Eur J Surg Oncol 2015;41:421-425.

5. Hardiman KM, Cone M, Sheppard BC, Herzig DO. Disparities in the treatment of colon cancer in octogenarians. Am J Surg 2009;197:624-628.

6. Golfinopoulos V, Pentheroudakis G, Pavlidis N. Treatment of colorectal cancer in the elderly: a review of the literature. Cancer Treat Rev 2006;32:1-8.

7. Nakamura T, Sato T, Miura H, et al. Feasibility and outcomes of surgical therapy in very elderly patients with colorectal cancer. Surg Laparosc Endosc Percutan Tech 2014;24:85-88.

8. Gurevitch AJ, Davidovitch B, Kashtan H. Outcome of right colectomy for cancer in octogenarians. J Gastrointest Surg 2009;13:100-104.

9. Yap R, Oliva K, Wilkins S, McMurrick PJ. Colorectal cancer surgery in the very elderly: nonagenarians. Dis Colon Rectum 2016;59:501-507.

10. Iversen LH, Pedersen L, Riis A, Friis S, Laurberg S, Sorensen HT. Age and colorectal cancer with focus on the elderly: trends in relative survival and initial treatment from a Danish population-based study. Dis Colon Rectum 2005;48:1755-1763.

11. Nascimbeni R, Di Fabio F, Di Betta E, Salerni B. The changing impact of age on colorectal cancer surgery: a trend analysis. Colorectal Dis 2009;11:13-18.

12. Kumar R, Jain K, Beeke C, et al. A population-based study of metastatic colorectal cancer in individuals aged $\geq 80$ years: findings from the South Australian Clinical Registry for Metastatic Colorectal Cancer. Cancer 2013;119:722-728.

13. Doat S, Thiebaut A, Samson S, Ricordeau P, Guillemot D, Mitry E. Elderly patients with colorectal cancer: treatment modalities and survival in France. National data from the ThInDiT cohort study. Eur J Cancer 2014;50:1276-1283.

14. McKibbin T, Frei CR, Greene RE, Kwan P, Simon J, Koeller JM. Disparities in the use of chemotherapy and monoclonal antibody therapy for elderly advanced colorectal cancer patients in the community oncology setting. Oncologist 2008;13:876-885. 
15. Seal BS, Sullivan SD, Ramsey SD, et al. Systemic therapy for colorectal cancer: patterns of chemotherapy and biologic therapy use in nationally representative US claims database. BioDrugs 2014;28:229-236.

16. Douillard JY, Hoff PM, Skillings JR, et al. Multicenter phase III study of uracil/tegafur and oral leucovorin versus fluorouracil and leucovorin in patients with previously untreated metastatic colorectal cancer. J Clin Oncol 2002;20:3605-3616.

17. Hoff PM, Ansari R, Batist G, et al. Comparison of oral capecitabine versus intravenous fluorouracil plus leucovorin as first-line treatment in 605 patients with metastatic colorectal cancer: results of a randomized phase III study. J Clin Oncol 2001;19:2282-2292.

18. Feliu J, Escudero P, Llosa F, et al. Capecitabine as first-line treatment for patients older than 70 years with metastatic colorectal cancer: an oncopaz cooperative group study. J Clin Oncol 2005;23:3104-3111.

19. Kroep JR, van Werkhoven E, Polee M, et al. Randomised study of tegafur-uracil plus leucovorin versus capecitabine as first-line therapy in elderly patients with advanced colorectal cancer: TLC study. J Geriatr Oncol 2015;6:307-315.

20. Sanoff HK, Goldberg RM. How we treat metastatic colon cancer in older adults. J Geriatr Oncol 2013;4:295-301.

21. Bradley CJ, Yabroff KR, Warren JL, Zeruto C, Chawla $\mathrm{N}$, Lamont EB. Trends in the treatment of metastatic colon and rectal cancer in elderly patients. Med Care 2016:54:490-497.

22. Aparicio T, Lavau-Denes S, Phelip JM, et al. Randomized phase III trial in elderly patients comparing $\mathrm{LV}_{5} \mathrm{FU}_{2}$ with or without irinotecan for first-line treatment of metastatic colorectal cancer (FFCD 2001-02). Ann Oncol 2016;27:121-127.

23. Kawakami K, Nakamoto E, Yokokawa T, et al. Patients' self-reported adherence to capecitabine on XELOX treatment in metastatic colorectal cancer: findings from a retrospective cohort analysis. Patient Prefer Adherence 2015;9:561-567.

24. Seal BS, Anderson S, Shermock KM. Factors associated with adherence rates for oral and intravenous anticancer therapy in commercially insured patients with metastatic colon cancer. J Manag Care Spec Pharm 2016;22:227-235.

25. Ohnishi T, Kanoh T, Kimura Y, et al. Successful low-dose TS-1 administration in an elderly colon cancer patient with liver metastasis. Gan To Kagaku Ryoho 2007;34:623625 .

26. Jung YH, Lee WJ, Byeon JH, Lee IK, Han CW, Woo IS. Metronomic chemotherapy with capecitabine for metastatic colorectal cancer in very elderly patients. Korean J Intern Med 2017;32:926-929.

27. Romiti A, Onesti CE, Roberto M, et al. Continuous, lowdose capecitabine for patients with recurrent colorectal cancer. Med Oncol 2015;32:54.

28. Rosati G, Aprile G, Cardellino GG, Avallone A. A review and assessment of currently available data of the EGFR antibodies in elderly patients with metastatic colorectal cancer. J Geriatr Oncol 2016;7:134-141.

29. Sclafani F, Cunningham D. Bevacizumab in elderly patients with metastatic colorectal cancer. J Geriatr Oncol 2014;5:78-88. 